\title{
Numerical simulations of two-way coupled particle laden mixing layers
}

\author{
E. Wallner* and E. Meiburg \\ Department of Aerospace and Mechanical Engineering \\ University of Southern California \\ Los Angeles, CA 90089-1191, USA \\ e-mail: eckart@spock.usc.edu \\ *Present address: \\ Institute of Flight Mechanics and Flight Control \\ University of Stuttgart \\ Pfaffenwaldring 7a, 70550 Stuttgart, Germany \\ e-mail: ewallner@ifr.luftfahrt.uni-stuttgart.de
}

\begin{abstract}
Navier-Stokes simulations are presented for two-way coupled, particle laden, two-dimensional mixing layers in the dilute regime. In contrast to previous numerical approaches for two-way coupled two-phase flows, the simulations are based on the vorticity, streamfunction formulation of the governing equations. While the fluid phase is treated in an Eulerian way, the particles are tracked in a Lagrangian fashion. The numerical approach is validated against simple test problems. Furthermore, the production/destruction of vorticity is shown to be related to the misalignment of the concentration gradient and the slip velocity, as well as to the difference in fluid and particle vorticities.
\end{abstract}

\section{Introduction}

Much insight into the evolution of particle laden mixing layers has been gained on the basis of one-way coupled numerical simulations, i.e., computations in which the fluid flow is not affected by the particle motion (Chein and Chung 1988, Martin and Meiburg 1994, Raju and Meiburg 1995, Marcu and Meiburg 1996a,b, Marcu et al. 1995, 1996, Crowe et al. 1996, and references therein). However, as the mass loading of the particular phase increases, it can substantially alter the evolution of the fluid flow. Such two-way coupling effects have been studied for isotropic and homogenous turbulence (Squires and Eaton 1990, Elghobashi and Truesdell 1993, Truesdell and Elghobashi 1994), as well as for wall turbulence (Pan and Banerjee 1996) and fully developed channel flows (Kulick et al. 1994). Further investigations by Druzhinin (1995), Park et al. (1996), and Ory et al. (1998) focus on cellular flow fields, jets, and mixing layers, respectively. The preferred approach for computationally implementing the particular phase has been Lagrangian in nature, i.e., in these simulations typically many particles are tracked individually on the basis of an equation that balances the forces acting on each of the particles. This approach is advantageous compared to solving a field equation for the particle concentration because of the particle inertia, which prevents the particle velocity from being a continuous, single-valued function of space and time. Consequently, the particle concentration would have to be solved for in phase space, cf. for 
example the spray equation derived by Williams (1964). This, in turn, is exceedingly expensive computationally due to the added dimensions of the problem. In the present investigation, we aim at refining the Lagrangian particle tracking approach, so that it will allow us the simulation of a continuous particle concentration field. In this way, we expect to obtain a tool that will allow us to identify more clearly the relevant mechanisms by which the particular phase affects the nonlinear evolution of the fluid motion in a mixing layer.

Considerable effort in the past has focused on examining the effects of a particulate phase on the stability of fluid flows. An early analytical study of steady laminar flows involving two-way coupling effects was carried out by Saffman (1961). He finds that fine particles with a small relaxation time relative to the characteristic time scale of the flow destabilize the fluid motion, while coarser ones with a greater relaxation time act stabilizing. Here, the particle concentration is assumed to be constant everywhere in the flow field. Chen and Chung (1995) confirm these findings for oscillatory two-phase channel flow by numerically solving a linearized stability equation in the limit of very small particles. The stability of two-way coupled particle laden mixing layers was first adressed by Yang et. al (1990). Their linear stability analysis, which neglects the particle response to flow fluctuations, reveals the stabilizing effect of particles on the spatial and temporal instabilities of a tanh shear layer. Wen and Evans (1994) focus on the effects of differential particle loading on the stability of the mixing layer. Assuming unresponsive particles, their linear stability analysis shows two coexisting unstable modes, one of which is the Kelvin-Helmholtz mode. The second mode is found to be similar to the Holmboe instability observed in density-stratified flows.

Very recently, Dimas and Kiger (1998) extended the above linear stability analyses by retaining particle dynamics. Their detailed investigation focuses on the impact of the particle Stokes number and the mass loading, and they observe that an increase in either one of these parameters decreases the growth rate of the instability. Under certain conditions, they find the appearance of a second, low-frequency instability mode in addition to the fundamental mode. Their results show the velocity, particle concentration, and vorticity fields to be strongly coupled.

Few experimental investigations specifically address the effects of two-way coupling. For a particle laden free jet, Fleckhaus et al. (1987) show that a mass loading of $30 \%$ has a considerable effect on the properties of the jet. Laser velocimetry experiments indicate that the centerline air velocity declines at a rate somewhat lower than that of the corresponding single phase jet. Kiger and Lasheras (1997) find that the dissipation in a two-phase turbulent mixing layer increases due to the particles' presence. We have been unable to find any experimental data on the effects of the particular phase on the growth rate and/or phase velocity of the Kelvin-Helmholtz instability in a mixing layer.

Considerable experimental and computational effort has been devoted towards an improved understanding of the mechanisms by which large scale vortical structures in mixing layers disperse heavy particles (Chein and Chung 1988, Lazaro and Lasheras 1989, 1992a,b, Martin and Meiburg 1994, Kiger and Lasheras 1995, Raju and Meiburg 1995, Marcu and Meiburg 1996a,b, Marcu et al. 1996). These investigations show intermediate particle sizes to undergo maximum dispersion. The ejection of particles from the vortex cores and the formation of high concentration particle streaks in the braid region are identified as crucial ingredients in the dispersion process. The present investigation then aims at identifying the ways in which two-way coupling effects modify these dispersion mechanisms. 


\section{Governing equations}

This investigation addresses the two-dimensional, temporal evolution of a particle-laden, viscous mixing layer, subject to two-way coupling effects. Assuming a dilute suspension of small, heavy, identical spherical particles, we employ a simplified form of the equation of motion derived by Maxey and Riley (1983) for spherical particles

$$
\begin{aligned}
m_{p} \frac{d \mathbf{u}_{p k}}{d t} & =6 \pi \mu a\left(\mathbf{u}_{\mathbf{k}}-\mathbf{u}_{\mathbf{p k}}\right) \\
\frac{d \mathbf{x}_{\mathbf{p k}}}{d t} & =\mathbf{u}_{\mathbf{p k}}
\end{aligned}
$$

It expresses the balance of particle inertia and viscous drag, while neglecting pressure, Basset history, virtual mass, and gravity forces, along with the Faxen corrections. If the density of the particle material is much greater than that of the fluid, then the terms in the Maxey equation due to virtual mass, pressure gradient and Basset history become negligible compared to the contribution of the viscous drag forces. By assuming creeping flow around the particles, we also neglect pressure drag effects. $m_{p}$ represents the mass of a particle, $\mu$ denotes the dynamic fluid viscosity, $a$ is the particle radius, and $\mathbf{x}_{p k}$ and $\mathbf{u}_{p k}$ indicate the location and velocity of particle $k$, respectively. $\mathbf{u}_{k}$ is the fluid velocity at the particle location.

In order to determine the collective force $\mathbf{f}$, with which the particles located in a differential control volume act on the fluid, we define the average particle velocity $\overline{\mathbf{u}}_{\mathbf{p}}$ in the differential control volume. In this way, we obtain

$$
\mathbf{f}=n 6 \pi \mu a\left(\overline{\mathbf{u}}_{\mathbf{p}}-\mathbf{u}\right)
$$

where $n$ denotes the particle number density. By neglecting the volume fraction of the particle phase in the continuity equation, the mass and momentum equations governing the constant density fluid motion take the form

$$
\begin{aligned}
\nabla \cdot \mathbf{u} & =0 \\
\rho \frac{\partial \mathbf{u}}{\partial t}+\rho(\mathbf{u} \cdot \nabla) \mathbf{u} & =-\nabla p+\mu \nabla^{\mathbf{2}} \mathbf{u}+n 6 \pi \mu a\left(\overline{\mathbf{u}}_{\mathbf{p}}-\mathbf{u}\right)
\end{aligned}
$$

Neglecting the volume fraction of the particle phase is a common assumption. If we take the density of the particle material to be several orders of magnitude higher than that of the fluid then for moderate mass loadings the volume fraction of the particles becomes negligible.

The particle and fluid equations are rendered dimensionless by introducing characteristic scales for velocity, length, and particle number density, respectively

$$
u^{*}=\Delta U, l^{*}=\delta_{m}, n^{*}=n_{0} .
$$


Here $\Delta U$ represents the velocity difference across the shear layer. $\delta_{m}$ is the width of the mixing layer at the initial time of the simulation, which serves as a characteristic length scale for the evolving vortical structure. $n_{0}$ indicates a nominal number density of the particles. The dimensionless governing equations thus are

$$
\begin{aligned}
\nabla \cdot \mathbf{u} & =0 \\
\frac{\partial \mathbf{u}}{\partial t}+(\mathbf{u} \cdot \nabla) \mathbf{u} & =-\nabla p+\frac{1}{R e} \nabla^{\mathbf{2}} \mathbf{u}+n \frac{D}{S t}\left(\overline{\mathbf{u}}_{\mathbf{p}}-\mathbf{u}\right), \\
\frac{d \mathbf{u}_{p k}}{d t} & =\frac{1}{S t}\left(\mathbf{u}_{k}-\mathbf{u}_{p k}\right), \\
\frac{d \mathbf{x}_{p k}}{d t} & =\mathbf{u}_{p k} .
\end{aligned}
$$

As the governing parameters, we identify the Reynolds number $R e$, along with the Stokes number St

$$
R e=\frac{\Delta U \delta_{m}}{\nu}, S t=\frac{\tau_{p}}{\tau_{f}}
$$

where the fluid time scale $\tau_{f}$ and the particle aerodynamic response time $\tau_{p}$ are defined as $\tau_{f}=$ $\delta_{m} / \Delta U$ and $\tau_{p}=2 a^{2} \rho_{p} / 9 \mu$, respectively. Here $\rho_{p}$ indicates the density of the particle material. $\tau_{p}$ can be viewed as a measure of the responsiveness of a particle to a change in the fluid velocity field, while $\tau_{f}$ gives an indication of the time available for the interaction of the particle with the large scale structures. The third parameter is the dimensionless mass loading $D$

$$
D=\frac{\bar{\rho}_{p}}{\rho}, \bar{\rho}_{p}=m_{p} n_{0}
$$

with $\bar{\rho}_{p}$ denoting the mass of particle material per unit volume of the flow.

For computational purposes, we formulate the two-dimensional equations governing the fluid motion in the streamfunction $(\psi)$ and vorticity $(\omega)$ variables, which yields

$$
\begin{aligned}
\frac{\partial \omega}{\partial t}+(\mathbf{u} \cdot \nabla) \omega & =\frac{1}{R e} \nabla^{\mathbf{2} \omega}+\frac{D}{S t} \nabla \times\left(n\left(\overline{\mathbf{u}}_{\mathbf{p}}-\mathbf{u}\right)\right) \cdot \mathbf{e}_{\mathbf{z}}, \\
\nabla^{\mathbf{2}} \psi & =-\omega .
\end{aligned}
$$

The vorticity equation allows us to identify physical mechanisms by which the particles may affect the mixing layer. The influence of the particulate phase, as represented by the source term, depends both on the mass loading as well as on the particle Stokes number. In addition, gradients in the particle number density and the velocity difference (slip velocity) between the two phases are expected to be of importance. While we expect larger mass loadings $D$ to result in stronger two-way coupling effects, the interpretation of the influence of $S t$ requires more caution, as this parameter also appears in the particle equation of motion, where it directly affects the slip velocity. 
The flow configuration is a two-dimensional shear layer with an initial velocity profile given by $u(y)=0.5 \tanh (y)$. The flowfield is periodic in the streamwise direction, while slip walls are applied at the upper and lower boundaries. For all simulations, the value of $R e$ is held fixed at 200. An initial velocity perturbation is applied that has the form of the eigenfunction of the most unstable mode according to inviscid theory as found by Michalke (1964). Its wavenumber is $\alpha=0.4446$, so that the length of the computational domain is $L=\frac{2 \pi}{\alpha}$. Here, we will consider only the case in which the entire flowfield is initially seeded uniformly with particles. At the beginning of the simulation, the particle velocities are equal to the respective fluid velocities at the particle locations.

\section{$3 \quad$ Numerical approach}

The field equations for the fluid motion are solved in an Eulerian fashion, by applying a spectral Fourier series expansion in the streamwise, periodic direction (Gottlieb and Orszag 1977), and sixth order compact finite differences in the transverse direction (Lele 1992). Time advancement of both the fluid and the particle equations is accomplished by means of a third order Runge-Kutta method (Wray 1991).

The dispersed particles are tracked in a Lagrangian way, with each of the $N$ computational particles representing a cluster of many physical particles located in the same neighborhood. The number density field $n(\mathbf{x}, t)$ is obtained as

$$
n(\mathbf{x}, t)=\sum_{i=1}^{N} \Gamma_{i} \gamma_{i}\left[\mathbf{x}-\mathbf{x}_{i}(t)\right]
$$

Here $\gamma_{i}$ represents the shape function of the number density distribution within the computational particle, normalized so that

$$
\int \gamma_{i}(\mathbf{x}) d \mathbf{x}=1
$$

In the present investigation, we take $\gamma_{i}$ to be radially symmetric and of Gaussian form

$$
\gamma_{i}(r)=\frac{1}{\pi \sigma_{i}^{2}} e^{-\frac{r^{2}}{\sigma_{i}^{2}}}
$$

where $\sigma_{i}$ is a measure of the core size of the computational cluster. $\Gamma_{i}$ denotes the 'strength' of cluster $i$, i.e., the overall number of particles contained in it. The numerical advantage of assuming a Gaussian distribution, which is commonly done in many Lagrangian computational procedures, is that we obtain a relatively smooth particle concentration field. If at time $t=0$ the clusters are separated by a distance $\Delta$ in the $x$ - and $y$-directions, we obtain

$$
\Gamma_{i}=\Delta^{2} n\left(\mathbf{x}_{i}, 0\right)
$$


In the numerical simulations to be discussed below, all computational clusters are of identical size and strength. Their initial separation $\Delta$ is typically equal to the grid size of the Eulerian mesh, while their core size $\sigma=2 \Delta$. In this way, there is sufficient overlap between the particle clusters to ensure a smooth number density field. In order to maintain a well-resolved particle concentration field for long times, a remeshing procedure for the particles is employed which inserts an additional cluster if the distance between two neighbors exceeds a certain value. The strengths $\Gamma_{i}$ of the two original clusters and the new cluster are then adjusted in order to maintain the overall number of particles in the flowfield. If the original neighbors had strengths $\Gamma_{1}^{*}$ and $\Gamma_{2}^{*}$ before the remeshing, their new strengths are determined as $\Gamma_{1}=\frac{3}{4} \Gamma_{1}^{*}$ and $\Gamma_{2}=\frac{3}{4} \Gamma_{2}^{*}$. The new cluster, which is introduced halfway between the original ones, and with their average velocity, is given the strength $\Gamma_{3}=\frac{1}{4}\left(\Gamma_{1}^{*}+\Gamma_{2}^{*}\right)$. In order to evaluate the particle equation of motion, we determine the flow velocity at the particle location by means of a fourth-order, two-dimensional Lagrangian interpolation scheme (Martin and Meiburg 1994). Particles that leave the control volume in the horizontal direction are reintroduced at the opposite boundary, in accordance with the periodic boundary conditions in the streamwise direction.

An important point concerns the time-dependent modification of the streamfunction boundary conditions at the upper and lower boundaries due to the two-way coupling effects. While our initial condition is such that there is no net fluid flow in the $x$-direction, this does not necessarily remain so for all times, as there can be a net $x$-momentum transfer from the particles to the fluid. Formulated differently, the momentum transfer from the particles to the fluid can lead to an overall acceleration of the fluid in the streamwise direction. This may happen if e.g. only a half-stream is initially seeded with particles. As a result, we have to account for a potential component $U$ of the fluid velocity in the $x$-direction. $U$ can be obtained as

$$
\frac{d U}{d t}=\frac{D}{S t} \bar{s}_{x}
$$

where $\bar{s}_{x}$ denotes the $x$-component of the momentum source term, averaged over the entire flowfield. For simulations with a fundamental perturbation wavelength only, the flowfield is discretized by a $65 \times 89$ mesh. A typical value of the time step is $\Delta t=0.25$, which needs to be decreased, however, for smaller St.

\section{Validation}

In order to validate the numerical approach, it is applied to a case that can easily be solved analytically. Consider a fluid that is initially at rest everywhere. At $t=0$, the entire flowfield is seeded with particles of unit velocity in the $x$-direction. The governing equations yield for the $x$-velocity components of the fluid and particle velocities

$$
\begin{aligned}
u(t) & =\frac{D}{1+D}\left(1-e^{-\frac{1+D}{S t} t}\right), \\
u_{p}(t) & =\frac{1}{1+D}\left(D+e^{-\frac{1+D}{S t} t}\right) .
\end{aligned}
$$

Figure 1 presents a comparison of numerical results with the analytical solution for $S t=1$ and $D=1$. A $129 \times 177$ grid was used, with $\sigma=2 \Delta$ and $\Delta t=0.125$. The numerical and analytical 
fluid velocity values cannot be distinguished in the graph, whereas small differences in the particle velocities are still noticable. It was found that with further grid refinement, these differences uniformly decrease to zero.

A variant of Stokes' first problem serves as a second, more challenging test case. An infinite flat plate is impulsively accelerated from rest to a constant velocity $U$ in its own plane. Liu (1966) formulates the equations governing the temporal evolution of the fluid and particle phases above the plate. In our notation, they are

$$
\begin{aligned}
\frac{\partial u}{\partial t} & =\frac{1}{R e} \frac{\partial^{2} u}{\partial t^{2}}+\frac{D}{S t}\left(u_{p}-u\right) \\
\frac{\partial u_{p}}{\partial t} & =\frac{1}{S t}\left(u-u_{p}\right)
\end{aligned}
$$

For the parameter values of $R e=100, S t=1$, and $D=1$, and the boundary conditions $u(y=$ $0, t)=0.5$ and $u(y \rightarrow \infty, t)=0$, figure 2 compares numerical simulation results obtained by the present algorithm with accurate solutions of the above coupled ODEs. It can be seen that a grid size of $65 \times 79$ in combination with a time step of $\Delta t=0.25$ and a particle radius of $\sigma=2 \Delta$ leads to very good agreement.

\section{$5 \quad$ Results}

It is instructive to discuss the differences in the vorticity and concentration fields between the passive (one-way coupled) and the two-way coupled cases. For uniform seeding with particles of $S t=1$, and for $D=0.5$, and $R e=200$, significant differences are visible, cf. figure 3 at $t=37.5$. Both in the braids and in the regions to the left and the right of the core, an increase in the magnitude of the vorticity can be observed. In contrast, the vorticity within the core, and in regions above and below it, is reduced in magnitude. Furthermore, the braids remain thicker under two-way coupling, and the vortex generally is less circular than in the passive case.

The particle concentration field is affected by the two-way coupling as well. As the coupling reduces the core vorticity, the centrifugal forces acting on the particles become weaker. As a result, for two-way coupling we observe an increase in particle concentration in the core, with a corresponding decrease in the braids. Due to the weaker ejection of particles from the core, the concentration field appears somewhat compressed in the cross-stream direction, when compared to the passive case. This behavior was also reported by Dimas and Kiger (1998) on the basis of their linear stability analysis.

A more detailed understanding of the underlying vorticity production and destruction mechanisms due to particle loading can be obtained by analyzing the source term in the vorticity equation. By expanding

$$
\frac{D}{S t} \nabla \times\left(n\left(\overline{\mathbf{u}}_{\mathbf{p}}-\mathbf{u}\right)\right)=\frac{D}{S t}\left(\nabla n \times\left(\overline{\mathbf{u}}_{\mathbf{p}}-\mathbf{u}\right)+n\left(\nabla \times \overline{\mathbf{u}}_{\mathbf{p}}-\nabla \times \mathbf{u}\right)\right),
$$

we identify two potential mechanisms for vorticity generation. The first term indicates that the vorticity will be altered if the concentration gradient is misaligned with the direction of the slip 
velocity. The second term describes the influence of a difference between the particle and fluid vorticities. In the vicinity of the core, this difference is largest, due to the particles' inertia. Keeping in mind that the fluid vorticity is negative everywhere, the source term will thus be positive in the core, thereby reducing the fluid vorticity. In the braids, on the other hand, the concentration gradient term becomes more significant due to the high particle accumulation. In combination with the local slip velocities, it leads to both production and destruction of vorticity. Overall, we find that the vorticity generation caused by two-way coupling tends to weaken the original Kelvin-Helmholtz instability.

We are currently conducting a parametric study in order to quantify the two-way coupling effects on the vorticity and particle concentration fields, as a function of the governing dimensionless parameters. Results will be reported elsewhere.

\section{Acknowledgments}

The authors gratefully acknowledge funding by NASA's Marshall Space Flight Center, as well as the collaboration with the PVAMU/Rocketdyne CFD Institute. The simulations were partially carried out at the San Diego Supercomputer Center, which is funded by the National Science Foundation.

\section{References}

1 Chein, R. And Chung, J.N.: Simulation of particle dispersion in a two-dimensional mixing layer. AIChE J. 34 (1988), 946-954.

2 Chen, Y.-C. And Chung, J.N.: The linear stability of an oscillatory two-phase channel flow in the limit of small Stokes number. Phys. Fluids 7 (1995), 1510-1512.

3 Crowe, C.T., Troutt, T.R., And Chung, J.N.: Numerical models for two-phase turbulent flows. Ann. Rev. Fluid Mech. 28 (1996), 11-43.

4 Dimas, A.A. AND Kiger, K.T.: Linear instability of a particle-laden mixing layer with a dynamic dispersed phase. Phys. Fluids 10 (1998), 2539-2557.

5 Druzhinin, O.A.: Dynamics of concentration and vorticity modification in a cellular flow. Phys. Fluids 7 (1995), 2132-2142.

6 Elghobashi, S. And Truesdell, G.C.: On the two-way interaction between homogeneous turbulence and dispersed solid particles. I: Turbulence modification. Phys. Fluids A 5 (1993), 1790-1801.

7 Fleckhaus, D., Hishida, K., And Maeda, M.: Effect of laden solid particles on the turbulent flow structure of a round free jet. Exp. in Fluids 5 (1987), 323-333.

8 Gottlieb, D. And Orszag, S.A.: Numerical Analysis of Spactral Methods: Theory and Applications. (1977) SIAM, Philadelphia.

9 Kiger, K.T. And Lasheras, J.C.: The effect of vortex pairing on the particle dispersion and kinetic energy transfer in a two-phase turbulent shear layer. J. Fluid Mech. 302 (1995), 149-178.

10 Kiger, K.T. And Lasheras, J.C.: Dissipation due to particle/turbulence interaction in a two-phase, turbulent, shear layer. Phys. Fluids 9 (1997), 3005-3023.

11 sc Kulick, J.D., Fessler, J.R., and Eaton, J.K.: Particle response and turbulence modification in fully developed channel flow. J. Fluid Mech. 277 (1994), 109-134.

12 Lazaro, B.J. And Lasheras, J.C.: Particle dispersion in a turbulent, plane, free shear layer. Phys. Fluids A 1 (1992), 1035-1044.

13 Lazaro, B.J. And Lasheras, J.C.: Particle dispersion in the developing free shear layer. Part 1. Unforced flow. J. Fluid Mech. 235 (1992), 143-178.

14 Lazaro, B.J. And Lasheras, J.C.: Particle dispersion in the developing free shear layer. Part 2. Forced flow. J. Fluid Mech. 235 (1992), 179-221.

15 LELE, S.K.: Compact finite difference schemes with spectral-like resolution. J. Comput. Phys. 103

ESAIM: Proc., Vol. 7, 1999, 436 -439 
(1992), 16-42.

16 Liu, J.T.C.: Flow induced by an oscillating infinite flat plate in a dusty gas. Phys. Fluids 9 (1966), $1716-1720$

17 Marcu, B. And Meiburg, E.: The effect of streamwise braid vortices on the particle dispersion in a plane mixing layer. I. Equilibrium points and their stability. Phys. Fluids 8 (1996), 715-733.

18 Marcu, B. And Meiburg, E.: Three-dimensional features of particle dispersion in a nominally plane mixing layer. Phys. Fluids 8 (1996), 2266-2268.

19 Marcu, B., Meiburg, E., And Newton, P.: Dynamics of heavy particles in a Burgers vortex. Phys. Fluids 7 (1995), 400-410.

20 Marcu, B., Meiburg, E., and Raju, N.: The effect of streamwise braid vortices on the particle dispersion in a plane mixing layer. II. Nonlinear particle dynamics. Phys. Fluids 8 (1996), 734-753.

21 Martin, J.E. And Meiburg, E.: The accumulation and dispersion of heavy particles in forced twodimensional mixing layers. I. The fundamental and subharmonic case. Phys. Fluids 6 (1994), 1116-1131.

22 MaXey, M.R. AND Riley, J.J.: Equation of motion for a small rigid sphere in a nonuniform flow. Phys. Fluids 26 (1983), 883-889.

23 Michalke, A.: On the inviscid instability of the hyperbolic-tangent velocity profile. J. Fluid Mech. 19 (1964), 543-555.

24 Ory, E., JoiA, I.A., And Perkins, R.J.: On the two-way interaction between a turbulent mixing layer and dispersed solid particles. Eur. Turb. Conf. (1998), 277-280.

25 PAN, Y. AND BANERJEe, S.: Numerical simulation of particle interactions with wall turbulence. Phys. Fluids 8 (1996), 2733-2755.

26 Park, T.W., Aggarwal, S.K., and Katta, V.R.: A numerical study of droplet-vortex interactions in an evaporating spray. Int. J. Heat Mass Transfer 39 (1996), 2205-2219.

27 Raju, N. And Meiburg, E.: The accumulation and dispersion of heavy particles in forced twodimensional mixing layers. Part 2: The effect of gravity. Phys. Fluids 7 (1995), 1241-1264.

28 Saffman, P.G.: On the stability of laminar flow of a dusty gas. J. Fluid Mech. 22 (1961), 120-128.

29 Squires, K.D. and Eaton, J.K.: Particle response and turbulence modification in isotropic turbulence. Phys. Fluids A 2 (1990), 1191-1203.

30 Truesdell, G.C. AND Elghobashi, S.: On the two-way interaction between homogeneous turbulence and dispersed solid particles. I: Particle dispersion. Phys. Fluids 6 (1994), 1405-1407.

31 Wen, F. And Evans, J.: Linear instability of a two-layer flow with differential particle loading. Phys. Fluids 6 (1994), 3893-3905.

32 Williams, F.A.: Combustion theory. Second edition (1985). Benjamin/Cummings.

33 Wray, A.A.: Minimal storage time-advancement schemes for spectral methods. Preprint (1991).

34 Yang, Y., Chung, J.N., Troutt, T.R., And Crowe, C.T.: The influence of particles on the spatial stability of two-phase mixing layers. Phys. Fluids A 2 (1990), 1839-1845. 


\section{Figures}

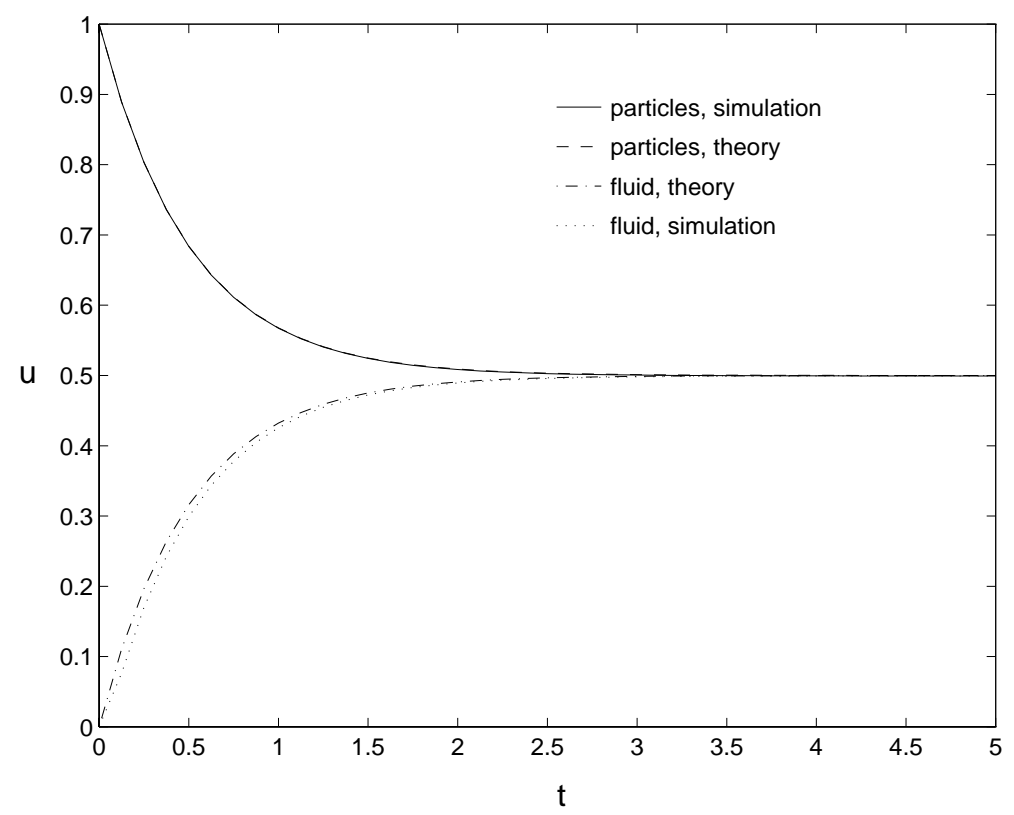

Figure 1: Comparison of analytical and numerical results obtained for a case where particles are uniformly distributed within the flowfield at an initial velocity $u_{p}(0)=1$ while the fluid is initially at rest. There is very good agreement for the fluid velocity. Differences in particle velocity decrease with further grid refinement.

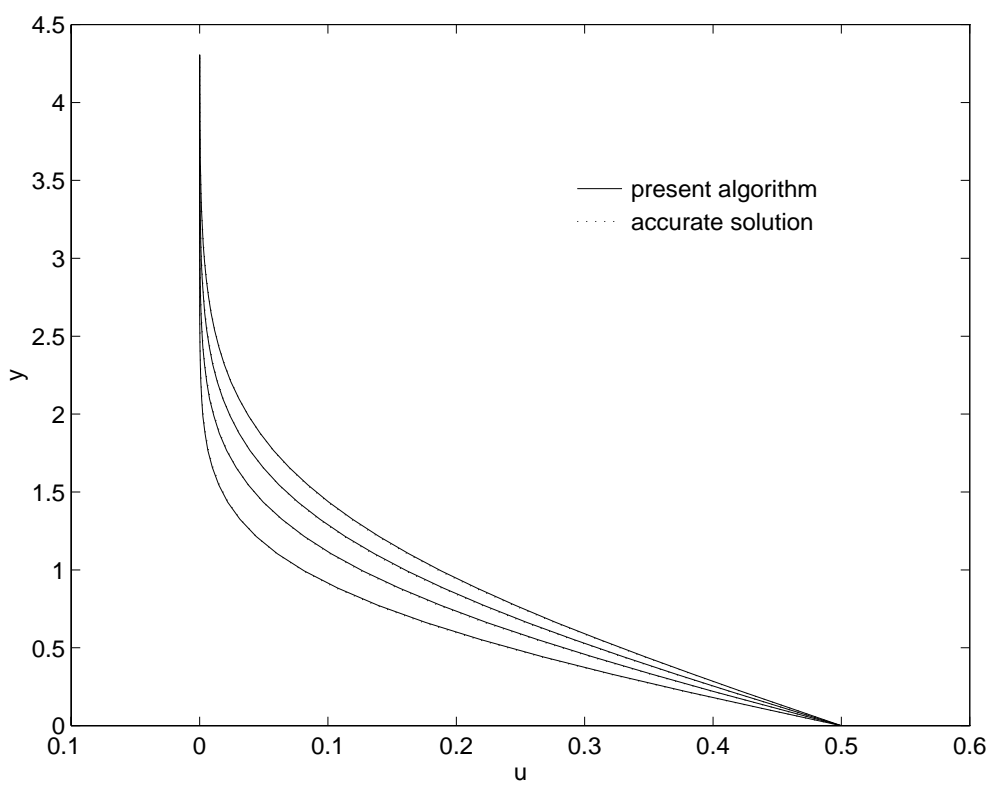

Figure 2: Result found for a variant of Stokes' first problem using present algorithm and, as a comparison, the accurate solution of the ODEs involved. The simulations were run for $R e=100, S t=1.0, D=1.0$. Velocity profiles are given for time $t=50,75,100,125$. Velocity of plate is $U=0.5$. Differences in the results obtained by the two programs are barely visible. 

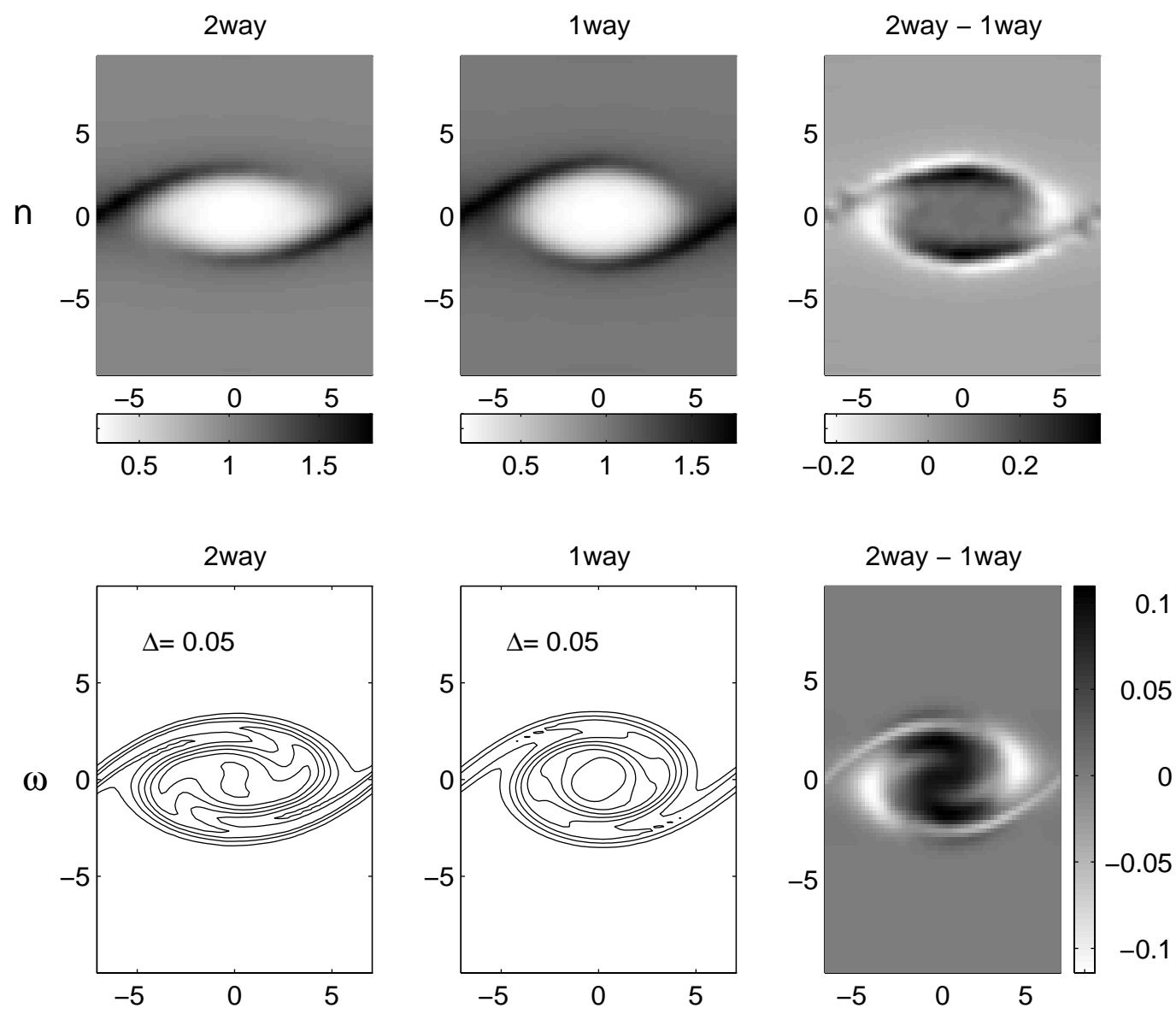

Figure 3: Particle concentration (top row) and vorticity fields (bottom row) for the coupled and passive cases, respectively. Plots on the righthand side show the differences between the two cases. Simulation parameters are: $S t=1, D=0.5, R e=200$. Time is $t=37.5$. In the vorticity contour plots (bottom row), $\Delta$ gives increments between contour levels. The difference in vorticity is shown by shading in the rightmost plot, where light shading visualizes an increase and dark shading visualizes a decrease in the magnitude of vorticity due to coupling. It can be seen that coupling leads to a decrease in vorticity in the core region and an increase in the braid region. As a result, the vortex loses its circular shape. As can be seen in the top row, particle-fluid interaction leads to an increase in particle concentration in the core region as visualized by the dark shading in the rightmost plot. 